\title{
ADDITIVE GROUPS OF RINGS WHOSE SUBRINGS ARE IDEALS
}

\author{
Shalom Feigelstock
}

\begin{abstract}
An Abelian group $G$ is called an $S I$-group if for every ring $R$ with additive group $R^{+}=G$, every subring $S$ of $R$ is an ideal in $R$. A complete description is given of the torsion $S I$-groups, and the completely decomposable torsion free $S I$-groups. Results are obtained in other cases as well.
\end{abstract}

All groups considered in this paper are Abelian, with addition the group operation. Rings are assumed to be associative, but need not possess a unity. Most of the results which will be obtained remain true for non-associative rings. $R^{+}$will denote the additive group of a ring $R$. The type function will be denoted by $t$. Both the ring and the additive group of integers and rational numbers will be denoted by $\mathbb{Z}$ and $\mathbb{Q}$ respectively. Terminology and notation will mostly follow $[3,4]$. One departure is the product of types in [4], which will be called the sum of types.

The object of this paper is to describe groups $G$ satisfying the following property: If $R$ is a ring with additive group $G$, then every subring of $R$ is an ideal in $R$.

Definition: A ring $R$ is an $S I$-ring if all of its subrings are ideals. A group $G$ is an $S I$-group if every ring $R$ with additive group $R^{+}=G$ is an $S I$-ring.

LEMMA 1. Let $R$ be a commutative ring with $R^{+}=G$, and let $M$ be an $R$ module, satisfying $R M \neq 0$, with $M^{+}=H$. Then $G \oplus H$ is not an $S I$-group.

PROOF: For $r_{1}, r_{2} \in R$, and $m_{1}, m_{2} \in M$ define $\left(r_{1}, m_{1}\right)\left(r_{2}, m_{2}\right)=\left(r_{1} r_{2}, r_{1} m_{2}\right.$ $\left.+r_{2} m_{1}\right)$. This multiplication induces a ring structure $S$ on $G \oplus H$. However the subring $T=\{(r, 0) \mid r \in R\}$ is not an ideal in $R$, because choosing $r \in R$, and $m \in M$ satisfying $r m \neq 0$, yields that $(r, 0)(0, m)=(0, r m) \notin T$.

COROLlaRY 2. For every Abelian group $H \neq 0$, the group $\mathbb{Z} \oplus H$ is not an SI-group.

Lemma 3. A direct summand of an $S I$-group is an $S I$-group.

Proof: Let $G=H \oplus K$, and suppose that there exists a non $S I$-ring $S$ with $S^{+}=$ $H$. Let $R$ be the ring with $R^{+}=G$, and multiplication defined by $\left(h_{1}, k_{1}\right)\left(h_{2}, k_{2}\right)=$

Received 27th June, 1996

Copyright Clearance Centre, Inc. Serial-fee code: 0004-9729/97 \$A2.00+0.00. 
$\left(h_{1} h_{2}, 0\right)$, where $h_{1} h_{2}$ is the product in $S$. Let $T$ be a subring of $S$ which is not an ideal in $S$, then $\{(t, 0) \mid t \in T\}$ is a subring of $R$ which is not an ideal in $R$.

It is well known, [3, Theorem 21.2], that a divisible subgroup of a group $G$ is a direct summand of $G$. This coupled with Lemma 3 yields:

COROLlaRY 4. If $\mathbb{Q}$ is a subgroup of $G$, then $G$ is not an $S I$-group.

An immediate consequence of Corollary 4 is:

Corollary 5. A torsion free SI-group is reduced.

A group $G$ is nil if the only ring $R$ satisfying $R^{+}=G$ is the ring with trivial multiplication, $R^{2}=0$.

Lemma 6. Let $p$ be a prime. A p-group $G$ is an $S I$-group if and only if either $G=Z\left(p^{n}\right), n$ a positive integer, or $G=D \oplus Z\left(p^{n}\right)$, with $D$ a divisible p-group, and $n=0$ or 1 .

Proof: Suppose that $G$ is an $S I$-group, and let $D$ be the maximal divisible subgroup of $G$. Then $G=D \oplus H$. If $H \neq Z\left(p^{n}\right), n$ a non-negative integer, then there exists positive integers $n \leqslant m$ such that $Z\left(p^{m}\right) \oplus Z\left(p^{n}\right)$ is a direct summand of $G,[3$, Corollary 27.3]. The ring $Z_{p^{m}}$ of integers modulo $p^{m}$ has additive group $Z\left(p^{m}\right)$, and

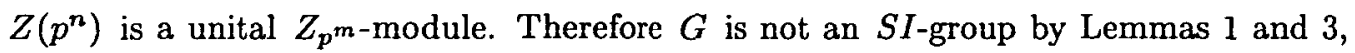
a contradiction. It may therefore be assumed that $G=D \oplus Z\left(p^{n}\right), n$ a non-negative integer, and it remains to be shown that if $D \neq 0$, then $n=0$ or $n=1$. If $D \neq 0$, then $H=Z\left(p^{\infty}\right) \oplus Z\left(p^{n}\right)$ is a direct summand of $G$, so by Lemma 3 , it suffices to show that $H$ is not an $S I$-group for $n>1$. Let $Z\left(p^{n}\right)=(a),|a|=p^{n}$, and let $d \in Z\left(p^{\infty}\right),|d|=p^{n}$. Defining multiplication in $H$ via $(x, k a)(y, m a)=(k m d, 0)$ for all $x, y \in Z\left(p^{\infty}\right)$, and all integers $k, m$, induces a ring structure $R$ on $H$. The subring $S$ of $R$ generated by $x=\left(p^{n-1} d, p^{n-1} a\right)$ is $S=\{k x \mid 0 \leqslant k<p\}$. Since $x \cdot(0, a)=\left(p^{n-1} d, 0\right) \notin S$, it follows that $S$ is not an ideal in $R$.

Conversely, if $G=Z\left(p^{n}\right), n$ a positive integer, then $G$ is clearly an $S I$-group. If $G$ is divisible, then $G$ is a nil group, [1, Theorem 2.1.1], and hence an $S I$-group. It remains to show that $G=D \oplus Z(p)$, with $D$ divisible, is an $S I$-group. Let $R$ be a ring with $R^{+}=G$, let $S$ be a rubring of $R$, and let $x_{0}=(0, a)$, with $|a|=p$. Since $R D=D R=0,[1, \mathrm{p} .8,1]$, it suffices to show that $x_{0} S \subseteq S$, and that $S x_{0} \subseteq S$. Since $D$ annihilates $R$, it follows that for $x_{1}=\left(d_{1}, m_{1} a\right), x_{2}=\left(d_{2}, m_{2} a\right) \in R$, the product $x_{1} x_{2}=m_{1} m_{2} x_{0}^{2}$. Therefore if $x_{0}^{2}=0$, then $x_{0} S=S x_{0}=0$, and $S$ is an ideal in $R$. If $x_{0}^{2} \neq 0$, then since $|a|=p$, it follows that $\left|x_{0}^{2}\right|=p$. Let $x=(d, m a) \in S$. If $m=0$, then $x_{0} \cdot x=x \cdot x_{0}=0$. It may therefore be assumed that $0<m<p$. Now $x^{2}=m^{2} x_{0}^{2}$. Since $p \nmid m$ there exists an integer $t$ such that $t m^{2}=1 \bmod p$. Therefore $x_{0}^{2}=t x^{2} \in S$, and so $x_{0} \cdot x=x \cdot x_{0}=m x_{0}^{2} \in S$.

It is well known, [3, Theorem 8.4], that a torsion group $G$ is the direct sum of its 
p-primary components, $G=\bigoplus_{p} G_{p}$. Similarly, a torsion ring $R$ is the ring direct sum of its $p$-primary components, $R=\bigoplus_{p} R_{p},[4, p .278(\mathrm{~B})]$. If $S$ is a subring of a torsion ring $R$, then $S_{p}=S \cap R_{p}$ for all primes $p$, and $R_{q} \cdot R_{p}=R_{p} \cdot R_{q}=0$ for all primes $q \neq p$. It therefore follows that a torsion group $G$ is an $S I$-group if and only if each of its $p$-primary components is an $S I$-group. This combined with Lemma 6 yields:

THEOREM 7. A torsion group $G$ is an SI-group if and only if $G=D \oplus$ $\bigoplus_{p \in P} Z\left(p^{n_{p}}\right)$, with $D$ a divisible torsion group, $P$ a set of distinct primes, $n_{p}$ a nonnegative integer for every $p \in P$, and $n_{p} \leqslant 1$ if $D_{p} \neq 0$.

LEMma 8. Let $G$ be an $S I$-group. Then $G_{p}$, the p-primary component of $G$, is a direct summand of $G$ for every prime $p$.

Proof: Let $D_{p}$ be the maximal divisible subgroup of $G_{p}$, then $G=D_{p} \oplus K$, [3, Theorem 21.2]. If $K_{p} \neq 0$ then, by [3, Corollary 27.3], $G=D_{p} \oplus Z\left(p^{n}\right) \oplus H$, with $n$ a positive integer. If $H_{p} \neq 0$ then, again by [3, Corollary 27.3], there exists a positive integer $m$ such that $Z\left(p^{n}\right) \oplus Z\left(p^{m}\right)$ is a direct summand of $G$. This contradicts Lemmas 3 and 6. Therefore $G_{p}=D_{p} \oplus Z\left(p^{n}\right)$ and so $G_{p}$ is a direct summand of $G$. D

It clearly follows from Lemma 8 and Lemma 3, that the p-primary component of an $S I$-group is an $S I$-group, for every prime $p$. This combined with the remarks preceding Theorem 7 yields:

Corollary 9. Let $G$ be an SI-group. Then $G_{t}$, the torsion part of $G$, is an SI-group.

THEOREM 10. Let $G$ be a mixed $S I$-group. Then $G_{t}=\bigoplus_{p \in P} Z\left(p^{n_{p}}\right)$, with $P$ a non empty set of distinct primes, and $n_{p}$ a positive integer for all $p \in P$.

Proof: By Corollary 9, and Theorem 7, it suffices to show that $D_{p}$, the maximal divisible subgroup of $G_{p}$, is trivial for every prime $p$. If not, then $G=Z\left(p^{\infty}\right) \oplus H$ for some prime $p$. Let $0 \neq a \in H$ be torsion free, and let $d \in Z\left(p^{\infty}\right)$, with $|d|=p^{2}$. There exists a homomorphism $\phi:(a) \oplus(a) \rightarrow Z\left(p^{\infty}\right)$ satisfying $\phi(a \otimes a)=d$. Since $Z\left(p^{\infty}\right)$ is injective in the category of Abelian groups, [3, Theorem 21.1], $\phi$ extends to a homomorphism $\phi: H \otimes H \rightarrow Z\left(p^{\infty}\right)$. The multiplication $\left(d_{1}, h_{1}\right)\left(d_{2}, h_{2}\right)=$ $\left(\phi\left(h_{1} \otimes h_{2}\right), 0\right)$ for all $d_{1}, d_{2} \in Z\left(p^{\infty}\right)$, and all $h_{1}, h_{2} \in H$, induces a ring structure $R$ on $G$. Since $(0, p a)(0, p a)=0$, it follows that the subring $S$ of $R$ generated by $(0, p a)$ is $S=\{(0, n p a) \mid(n \in \mathbb{Z})\}$. However $(0, a)(0, p a)=(p d, 0) \notin S$, and so $S$ is not an ideal in $R$.

CoRollary 11. Let $G$ be an $S I$-group, and let $p$ be a prime for which $G_{p} \neq 0$. Then $G=G_{p} \oplus H$, and $H$ is $p$-divisible.

Proof: $G_{p}$ is a direct summand of $G$ by Lemma 8 . It may be assumed by 
Theorem 10, that $G_{p}=(a)$, with $|a|=p^{n}$. Suppose that $H$ is not $p$-divisible. Then there exists $h \in H$ with zero $p$-height. $\bar{h}=h+p H$ has order $p$ in the group $\bar{H}=H / p H$, and $\bar{H}=(h) \oplus K$. Let $\phi:(a) \otimes \bar{H} \rightarrow(a)$ be the homomorphism induced by the maps $\phi[a \otimes(m \bar{h}, k)]=m a$, for all $0 \leqslant m<p$, and all $k \in K$. The multiplication $\left(m_{1} a, h_{1}\right)\left(m_{2} a, h_{2}\right)=\left(m_{1} \phi\left(a \otimes \bar{h}_{2}\right)+m_{2} \phi\left(a \otimes \bar{h}_{1}\right), 0\right)$ for all $0 \leqslant m_{1}, m_{2}<p^{n}$, and all $h_{1}, h_{2} \in H$, induces a ring structure $R$ on $G$. The subring $S$ of $R$ generated by $(0, h)$ is $=\{(0, n h) \mid n \in \mathbb{Z}\}$, but $(a, 0)(0, h)=(a, 0) \notin S$, and so $S$ is not an ideal in $R$.

An immediate consequence of Corollary 11 is:

Corollary 12. Let $G$ be an $S I$-group, and let $p$ be a prime such that $G_{p} \neq 0$. Then $G / G_{t}$ is $p$-divisible.

Lemma 13. Let $G=\bigoplus_{i \in I} G_{i}$. If there exist $i, j, k \in I$ such that $i \neq j, i \neq k$, and if there exists $\phi \in \operatorname{Hom}\left(G_{i} \otimes G_{j}, G_{k}\right)$ such that $\phi \neq 0$, then $G$ is not an $S I$-group.

Proof: Let $0 \neq \phi \in \operatorname{Hom}\left(G_{i} \otimes G_{j}, G_{k}\right)$. There are two cases to consider:

(1) $j \neq k$, and

(2) $j=k$.

It may be assumed that $i=1, j=2$, and $k=3$ in case (1), and that $i=1, j=k=2$ in case (2). By Lemma 3, it suffices to show that $H=G_{1} \oplus G_{2} \oplus G_{3}$ is not an $S I$-group in cae (1), and that $H=G_{1} \oplus G_{2}$ is not an $S I$-group in case (2). Let $a_{u} \in G_{u}, u=1,2$, such that $\phi\left(a_{1} \otimes a_{2}\right) \neq 0$. Let $R$ be the ring with $R^{+}=H$, and multiplication defined by $\left(b_{1}, b_{2}, b_{3}\right)\left(c_{1}, c_{2}, c_{3}\right)=\left(0,0, \phi\left(b_{1} \otimes c_{2}+c_{1} \otimes b_{2}\right)\right)$ for all $b_{u}, c_{u} \in G_{u}, u=1,2,3$ in case (1), and by $\left(b_{1}, b_{2}\right)\left(c_{1}, c_{2}\right)=\left(0, \phi\left(b_{1} \otimes c_{2}+c_{1} \otimes b_{2}\right)\right)$ for all $b_{u}, c_{u} \in G_{u}, u=$ 1,2 in case (2). Let $S$ be the subring of $R$ generated by $\left(a_{1}, 0,0\right)$ in case (1), and by $\left(a_{1}, 0\right)$ in case (2). Then $\left(a_{1}, 0,0\right)\left(0, a_{2}, 0\right)=\left(0,0, \phi\left(a_{1} \otimes a_{2}\right)\right) \notin S$ in case (1), and $\left(a_{1}, 0\right)\left(0, a_{2}\right)=\left(0, \phi\left(a_{1} \otimes a_{2}\right)\right) \notin S$ in case (2). In either case, $S$ is not an ideal in $R$.

The following two results concerning nil rank 1 , and completely decomposable torsion free groups will be useful in obtaining a description of the completely decomposable torsion free $S I$-groups.

Proposition 14. Let $G$ be a rank 1 torsion free group $G$. Then $G$ is nil if and only if $t(G)$ is not idempotent. If $G$ is not nil, then either $G \simeq \mathbb{Z}$, or $G$ is the additive group of a ring isomorphic to a subring of $\mathbb{Q}$ which contains $\mathbb{Z}$ as a proper subring.

Proof: $[1,1.4 .8]$, and $[4$, Theorem 121.1].

Proposition 15. Let $G=\bigoplus_{i \in I} G_{i}$ be completely decomposable, with each $G_{i}$ 
a rank 1 torsion free group. Then $G$ is nil if and only if $t\left(G_{i}\right)+t\left(G_{j}\right) \nless t\left(G_{k}\right)$ for all $i, j, k \in I$.

ProOF: [1, Corollary 2.1.3].

THEOREM 16. Let $G=\bigoplus_{i \in I} G_{i}$ be a completely decomposable torsion free group, with each $G_{i}$ a rank 1 group. Then $G$ is an $S I$-group if and only if either $G \simeq \mathbb{Z}$, or $G$ is nil.

Proof: Suppose that $G$ is an $S I$-group. If $G$ is a rank 1 group then Proposition 14 implies that either $G \simeq \mathbb{Z}$ or $G$ is nil. It may therefore be assumed that $G$ has rank greater than 1. It follows from Proposition 14, Corollary 2, and Lemma 3, that $t\left(G_{i}\right)$. is not idempotent, and so $t\left(G_{i}\right)+t\left(G_{i}\right) \notin t\left(G_{i}\right)$ for all $i \in I$. If there exist $i, j, k \in I$ such that $i \neq j, i \neq k$ and $t\left(G_{i}\right)+t\left(G_{j}\right) \leqslant t\left(G_{k}\right)$ then Hom $\left(G_{i} \otimes G_{j}, G_{k}\right) \neq$ 0 , [4, Propositions 85.3 and 85.4]. Lemma 13 yields that $G$ is not an $S I$-group, a contradiction. To show that $G$ is nil it suffices to show, by Proposition 15, that $t\left(G_{i}\right)+t\left(G_{i}\right) \nless t\left(G_{j}\right)$ for all $i, j \in I, i \neq j$. It may be assumed that $i=1, j=2$, and that $G_{u}$ is nil, $u=1,2$. Suppose that $t\left(G_{1}\right)+t\left(G_{1}\right) \leqslant t\left(G_{2}\right)$. By Lemma 3, it suffices to show $H=G_{1} \oplus G_{2}$ is not an $S I$-group. Since $t\left(G_{1}\right)$ is not idempotent, Proposition 14, there exists $a_{1} \in G_{1}$, and a prime $p$, such that the $p$-height if $a_{1}$ is 1 . There exists $0 \neq \phi \in \operatorname{Hom}\left(G_{1} \otimes G_{1}, G_{2}\right),[4$, Propositions 85.3 and 85.4]. Let $R$ be the ring with $R^{+}=H$, and multiplication defined by $\left(b_{1}, b_{2}\right)\left(c_{1}, c_{2}\right)=\left(0, \phi\left(b_{1} \otimes c_{1}\right)\right)$ for all $b_{u}, c_{u} \in G_{u}, u=1,2$. Let $S$ be the subring of $R$ generated by $(a, 0)$, and let $a_{0} \in G_{1}$ such that $p a_{0}=a$. Then $\left(a_{0}, 0\right)(a, 0)=\left(0, \phi\left(a_{0} \otimes a\right)\right) \notin S$, and so $S$ is not an ideal in $R$.

\section{REFERENCES}

[1] S. Feigelstock, Additive groups of rings, Research Notes in Mathematics 83 (Pitman, London, 1983).

[2] S. Feigelstock, Additive groups of rings II, Research Notes in Mathematics 169 (Longman, Harlow, 1988).

[3] L. Fuchs, Infinite Abelian groups 1 (Academic Press, New York, London, 1971).

[4] L. Fuchs, Infinite Abelian groups 2 (Academic Press, New York, London, 1973).

Department of Mathematics and Computer Science

Bar Ilan University

Ramat-Gan

Israel 